\title{
The atlas of aging society 2.0
}

\author{
Lina Dönni ${ }^{\text {a,*, Susanne Bleisch }}{ }^{\text {a }}$, Rainer Roth ${ }^{\text {a }}$, Mario Pozza ${ }^{\text {a }}$, Daria Hollenstein ${ }^{\text {a }}$, Alesya \\ Heymann $^{\mathrm{a}}$ \\ ${ }^{a}$ Institute of geomatics FHNW, Lina Dönni - lina.doenni@fhnw.ch, Susanne Bleisch - susanne.bleischi@fhnw.ch, Rainer Roth - \\ rainer.roth@fhnw.ch, Mario Pozza - mario.pozzai@fhnw.ch, Daria Hollenstein - daria.hollenstein@fhnw.ch, Alesya Heymann- \\ alesya.heymann@fhnw.ch \\ * Corresponding author
}

Keywords: atlas of the aging society 2.0, age-related information, storylines, story-network, interactive web application

\begin{abstract}
:
Most of the western countries currently experience a demographic change. It is essential catching this structural change with all its socio-spatial and life-specific dimensions and make it visible in a generally understandable way.

The atlas of the aging society 2.0, based on a first version launched in 2017, aims to visualize age-related information in an interactive web application that supports not only the content but also engages the users, is accessible to a broad audience, offers opportunities for different stakeholders and levels of interest, and can accommodate a high range of data as well as future updates.

As in the initial version, the atlas of aging society 2.0 operates with a novel concept called the story-network principle which connects briefly introduced visualizations such that a braid of age-related information develops. Like conventional atlases, the dimensions of information are divided into different topic areas. Each topic comprises several stories consisting of multiple information cards. Various connecting lines link the cards together and integrate all of them into an informational network. Storylines connect cards in a directional order such that their information covers a particular aspect of a topic. Related-card lines link cards that have similar content but do not belong to the same story, while recommended-card lines prevent users from dead ends by connecting story ending cards with different story starting cards. The user decides at any time whether he/she wants to follow a storyline through a whole topic aspect or if he/she interrupts the story and navigates into other topics to explore further information.
\end{abstract}

The story-network is visibly displayed on the web application's landing page and follows the reverse conclusion from Tobler's first law of geography. The similarity in the content of information cards reflects their spatial position in the network. The information cards are displayed as nodes. The appearance of the edges explains the type of connection between the nodes. To immerse oneself in the informational network of the atlas of aging society 2.0, different options are available. It is possible to navigate directly from the story-network view on the landing page to a specific card by a single click on the representative node. To give the user an idea of what information is hidden behind the nodes, the title of the nodes can be displayed in the story network view using an on-touch function. The topic-entry option is ideal for users who search for specific information. Therefore, an interactive and hierarchically organized bubble plot was created. When hovered, the bubble plot first divides into topic-area bubbles. On a second level, these are divided into individual stories, from which the user can choose. A third option is the usage of the coincidence-button, leading the user to a random card.

Each card includes a visualization, a descriptive text, and some additional information compromised in the metadata. The visualizations follow a uniform visual language, by using a constant colour concept and an overarching design language. The graphical representations use the D3.js and DC.js JavaScript libraries. One aim of the atlas of aging society 2.0 was to make visualizations more interactive by working with hover- and click-functions and by the implementation of interactive dashboards. The ability to interact with selected visualizations intends to motivate users to explore and display data according to their interests.

The atlas of aging society 2.0 is currently optimized for tablets while a desktop version is in progress. In this version, there are many new exciting subject areas and contents, which can be explored. The use of the story-network principle as implemented in this project is also conceivable for other applications due to its linking and ordering properties and its easy scalability. 